\title{
Joint resource allocation scheme for target tracking in distributed MIMO radar systems
}

\author{
ZHENG Na'e, SUN Yang, SONG Xiyu*, and CHEN Song
}

Data and Target Engineering Institute, PLA Strategic Support Force Information Engineering University, Zhengzhou 450001, China

\begin{abstract}
A joint resource allocation scheme concerned with the sensor subset, power and bandwidth for range-only target tracking in multiple-input multiple-output (MIMO) radar systems is proposed. By selecting an optimal subset of sensors with the predetermined size and implementing the power allocation and bandwidth strategies among them, this algorithm can help achieving a better performance within the same resource constraints. Firstly, the Bayesian Cramer-Rao bound (BCRB) is derived from it. Secondly, a criterion for minimizing the BCRB at the target location among all targets tracking in a certain range is derived. Thirdly, the optimization problem involved with three variable vectors is formulated, which can be simplified by deriving the relationship between the optimal power allocation vector and the bandwidth allocation vector. Then, the simplified optimization problem is solved by the cyclic minimization algorithm incorporated with the sequential parametric convex approximation (SPCA) algorithm. Finally, the validity of the proposed method is demonstrated with simulation results.
\end{abstract}

Keywords: distributed multiple-input multiple-output (MIMO) radar, target tracking, joint resource alloction, sensor subset selection (SSS), optimal power and bandwidth allocation (OPBA).

DOI: $10.21629 / J S E E .2019 .04 .09$

\section{Introduction}

The multiple-input multiple-output (MIMO) radar is rapidly expanding for its excellent performance in multitarget processing as well as target detecting against radar cross section (RCS) fluctuations [1]. There are two main MIMO radar configurations: colocated sensors and distributed sensors. The MIMO radar with colocated sensors can improve the estimated performance by making use of waveform diversity [2,3], while the MIMO radar with the distributed sensor takes advantage of the spatial diversity supported by the system configuration [4,5]. The distributed MIMO radar is selected in this paper, since it

\footnotetext{
Manuscript received June 04, 2018.

*Corresponding author.

This paper was supported by the National Natural Science Foundation of China (61501513; 61401469; 41301481).
}

outperforms the colocated MIMO radar in terms of the target tracking capability [6].

The research on resource allocation in the MIMO radar is drawing increasing attention between domestic and overseas scholars. In [6], the Cramer-Rao bound on the target location was derived for the MIMO radar. In [7], the Bayesian Cramer-Rao bound (BCRB) on target tracking was derived for evaluating the tracking performance, which illustrated the impact of the sensor location, target location, the propagation pathloss, the target reflectivity, the power and effective bandwidth on target tracking. Theoretically, more sensors and more transmit resources can achieve better accuracy for target localization and tracking. However, the MIMO radar is a multi-sensor system where the data fusion is a fundamental procedure for signal processing. Therefore, the redundant sensors will increase the complexity in central processing. Besides, the survival rate is a very important indicator to measure the performance of the radar system in hostile environments, which requires the resource management $[8,9]$.

Two main categories on resource allocation with the MIMO radar are evolved [10,11]: the first category is system structure optimizing based, such as the subset selection and placement of the sensors. The second category is signal parameters settings based, which includes the transmit power, bandwidth, etc. For the first category, two operational strategies were considered in [12], which were to select an optimal subset of active sensors for the best estimation performance or to make use of the minimum sensors to satisfy the predetermined estimation threshold. Furthermore, a joint transmitter and receiver selection problem was analyzed for multiple targets tracking in [13], where target priorities were considered. In the second category, the attainable localization mean square error (MSE) is minimized through power allocation among the sensors under the total power resource [14,15]. When the power and the bandwidth budget were limited, a suboptimal joint resource allocation strategy was proposed in [16], which 
achieved the optimal localization accuracy for multiple stationary targets. Moreover, a joint resource allocation scheme concerned with the sensor subset and power allocation was proposed in [17] to improve the target localization accuracy, which was converted into the second-order cone programming (SOCP) problem and solved by convex optimization. In [18], the optimal power allocation scheme was modeled as a cooperative game for the better localization performance of range-only target tracking, and it was solved by obtaining the Shapley value.

In addition, the target tracking performance is also related to the sensor performance and the signal bandwidth. Furthermore, the bandwidth allocation strategy is more important than power allocation for a better localization performance [16]. The resource allocation approaches proposed in [13 - 16] aim at improving the single/multiple stationary targets localization accuracy, in which the coarse target locations are estimated by using the best linear unbiased estimation (BLUE) method. The coarse estimation is served as the input of the resource allocation algorithms. However, the target location cannot be obtained previously. It is more practical for MIMO radar systems to predict the target location next time according to the current observation data, i.e., the resource allocation is more suitable for target tracking [19,20].

In this paper, a joint resource allocation scheme concernd with the sensor subset, power as well as the signal bandwidth under resource constraints for a better accuracy of range-only target tracking is proposed. Firstly, the system model is established and the BCRB of the target location accuracy is given. Then, a joint resource allocation optimization model is fomulated to minimize the BCRB, which is involved with three different vectors. By deriving the relationship between two of the vectors, the complex optimization problem is effectively simplified and then solved by the cyclic minimization algorithm incorporated with the sequential parametric convex approximation (SPCA) algorithm [21].

The paper is organized as follows. Section 2 explains the system model. In Section 3, the objective function, i.e., the $\mathrm{BCRB}$, for evaluating the target tracking performance is derived. Section 4 presents the considered joint resource allocation problem, and discusses the simplified method as well as the solutions to the simplified optimization problem. Simulations and analysis of the proposed algorithm are provided in Sections 5. Finally, conclusions are addressed in Section 6.

\section{System model}

Considering that a widely separated MIMO radar system consists of $M$ transmitters and $N$ receivers, the po- sitions of the $m$ th transmit sensor and the $n$th receive sensor are denoted as $\left(x_{m}^{t}, y_{m}^{t}\right)^{\mathrm{T}}$ and $\left(x_{n}^{r}, y_{n}^{r}\right)^{\mathrm{T}}$, respectively, where $m \in M, n \in N$. The low-pass equivalence of the $m$ th transmitted waveform is denoted as $s_{m}(t)$, $\int_{T_{m}}\left|s_{m}(t)\right|^{2} \mathrm{~d} t=1$, where $T_{m}$ is the duration time. The single target moves with the initial location $\left(x_{0}, y_{0}\right)^{\mathrm{T}}$ and velocity $\left(\dot{x}_{0}, \dot{y}_{0}\right)^{\mathrm{T}}$. For the resource management, we define the power vector $\boldsymbol{p}^{k}=\left[p_{1}^{k}, \ldots, p_{M}^{k}\right]^{\mathrm{T}}$ and the effective bandwidths vector $\boldsymbol{\beta}^{k}=\left[\beta_{1}^{k}, \ldots, \beta_{M}^{k}\right]^{\mathrm{T}}$ at time $k$. To select the sensors, a binary variable vector $\boldsymbol{u}^{k}=$ $\left[u_{1}^{k}, u_{2}^{k}, \ldots, u_{M}^{k}\right]^{\mathrm{T}}$ is introduced, where $u_{m}^{k}=1$ indicates that the $m$ th transmitting sensor is selected at time $k$, otherwise $u_{m}^{k}=0 . \boldsymbol{\theta}_{k}=\left[x_{k}, y_{k}\right]^{\mathrm{T}} \in \mathbf{R}^{2}$ is the target position vector and moves with $\left(\dot{x}_{k}, \dot{y}_{k}\right)^{\mathrm{T}}$ at time $k$, which is shown in Fig. 1. $d_{t, m}^{k}$ is the range from the $m$ th transmitter to the target, and $d_{r, n}^{k}$ is the range from the target to the $n$th receiver at time $k$. The time delay of the $(m, n)$ th path [12] can be caculated by

$$
\tau_{m n}^{k}=\frac{d_{t, m}^{k}+d_{r, n}^{k}}{c}
$$

where

$$
\left\{\begin{array}{l}
d_{t, m}^{k}=\sqrt{\left(x_{k}-x_{m}^{t}\right)^{2}+\left(y_{k}-y_{m}^{t}\right)^{2}} \\
d_{r, n}^{k}=\sqrt{\left(x_{k}-x_{n}^{r}\right)^{2}+\left(y_{k}-y_{n}^{r}\right)^{2}}
\end{array} .\right.
$$

$c$ is the light speed. $\phi_{m}^{k}$ and $\varphi_{n}^{k}$ are the angle from the $m$ th transmitter and the $n$th receiver to the target respectively, where

$$
\begin{aligned}
\phi_{m}^{k} & =\arctan \left[\left(y_{m}^{t}-y_{k}\right) /\left(x_{m}^{t}-x_{k}\right)\right], \\
\varphi_{n}^{k} & =\arctan \left[\left(y_{n}^{r}-y_{k}\right) /\left(x_{n}^{r}-x_{k}\right)\right] .
\end{aligned}
$$

The baseband signal transmitted from the $m$ th transmitter and received by the $n$th receiver at time $k$ [12] can be represented as

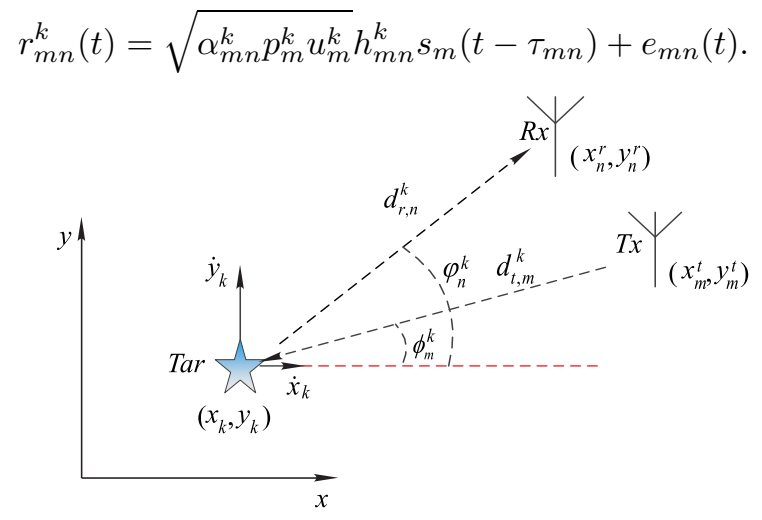

Fig. 1 Locations of the radar sensors and the target

The term $\alpha_{m n}^{k} \propto \frac{1}{\left(d_{t, m}^{k} d_{r, n}^{k}\right)^{2}}$ denotes the free space propagation factor. The corresponding target RCS is denoted as a known complex variable $h_{m n}^{k}$. The term $e_{m n}(t)$ 
denotes the circularly symmetric, zero-mean, complex Gaussian noise, spatially and temporally white with autocorrelation function $\sigma_{\omega}^{2} \delta(\tau)$.

\subsection{Target motion}

We consider that a target moves with a nearly constant velocity (CV). $\boldsymbol{x}_{k}=\left(x_{k}, y_{k}, \dot{x}_{k}, \dot{y}_{k}\right)^{\mathrm{T}}$ represents the target state at time $k$, including the target location and velocity. The target motion [7] can be expressed as

$$
\begin{gathered}
\boldsymbol{x}_{k+1}=\boldsymbol{F} \boldsymbol{x}_{k}+\boldsymbol{\omega}_{k}, \\
\boldsymbol{F}=\left[\begin{array}{cc}
\boldsymbol{I} & \Delta t \boldsymbol{I} \\
\mathbf{0} & \boldsymbol{I}
\end{array}\right]
\end{gathered}
$$

where $\Delta t$ is the observation time interval, and $\boldsymbol{\omega}_{k}$ is the plant noise, which is modeled as white Gaussian noise with covariance matrix $\boldsymbol{Q}_{k}$ :

$$
\boldsymbol{Q}_{k}=q \times\left[\begin{array}{cc}
\frac{1}{3} \Delta t^{3} \boldsymbol{I} & \frac{1}{2} \Delta t^{2} \boldsymbol{I} \\
\frac{1}{2} \Delta t^{2} \boldsymbol{I} & \Delta t \boldsymbol{I}
\end{array}\right]
$$

where $q$ is the intensity of the process noise, and $\boldsymbol{I}$ is a $2 \times 2$ dimension identity matrix.

\subsection{Measurement model}

Define $\boldsymbol{z}^{k}\left(\boldsymbol{u}^{k}, \boldsymbol{p}^{k}, \boldsymbol{\beta}^{k}\right)=\left[z_{11}^{k}, z_{12}^{k}, \ldots, z_{M N}^{k}\right]^{\mathrm{T}}$ as the range measurement vector at time $k$. In practice, the accurate range of the target is usually hard to obtain due to the measurement noise. Thus the measurement data received by the radar system is accompanied with the random error. The measurement model of the $(m, n)$ th transmit/receive channel [18] can be written as

$$
z_{m n}^{k}=d_{t, m}^{k}+d_{r, n}^{k}+e_{m n}^{k}
$$

where $e_{m n}^{k}$ is the measurement error, which is related to the signal to noise ratio (SNR) in the echo signal. The corresponding SNR [7] is defined as follows:

$$
\mathrm{SNR}_{m n}^{k}=\frac{\alpha_{m n}^{k} p_{m}^{k}\left|h_{m n}^{k}\right|^{2}}{\sigma_{\omega}^{2}} .
$$

The measurement error vector $\boldsymbol{e}_{z}^{k}=\left[e_{11}^{k}, e_{12}^{k}, \ldots\right.$, $\left.e_{M N}^{k}\right]^{\mathrm{T}}$ is Gaussian with zero mean and the error covariance matrix can be recast as

$$
\begin{aligned}
C_{z}^{k}= & \frac{c^{2}}{8 \pi^{2}} \operatorname{diag}\left(\frac{1}{\left(\beta_{1}^{k}\right)^{2} \mathrm{SNR}_{11}}, \frac{1}{\left(\beta_{1}^{k}\right)^{2} \mathrm{SNR}_{12}}, \ldots,\right. \\
& \left.\frac{1}{\left(\beta_{1}^{k}\right)^{2} \mathrm{SNR}_{1 N}}, \ldots, \frac{1}{\left(\beta_{M}^{k}\right)^{2} \mathrm{SNR}_{M N}}\right) .
\end{aligned}
$$

The fusion center deals with the measurement data after the sensor subset selection is finished,

$$
\boldsymbol{z}^{k}=\left[[1, \ldots, 1]_{N \times 1} \otimes \boldsymbol{u}^{k}\right]_{M N \times 1} \odot\left[h\left(\boldsymbol{x}_{k}\right)+\boldsymbol{e}_{z}^{k}\right]
$$

where $\otimes$ denotes the Kronecker product and $\odot$ denotes the Hadamard product between vectors. $h\left(\boldsymbol{x}_{k}\right)=\boldsymbol{r}\left(\boldsymbol{x}_{k}\right)$ is highly nonlinear, whose $(m n)$ th element $r_{m n}^{k}$ satisfies $r_{m n}^{k}=d_{t, m}^{k}+d_{r, n}^{k}$, in which $d_{t, m}^{k}$ and $d_{r, n}^{k}$ are defined in (2).

\section{BCRB}

For evaluating the target tracking performance, the BCRB was derived in [7]. Considering that an unknown vector $\boldsymbol{x} \in \mathbf{R}^{n \times 1}$ is estimated by using an observation vector $\boldsymbol{z}$, the corresponding BCRB [7] can be written as

$$
\mathrm{E}_{\boldsymbol{z}, \boldsymbol{x}}\left\{[\widehat{\boldsymbol{x}}(\boldsymbol{z})-\boldsymbol{x}][\widehat{\boldsymbol{x}}(\boldsymbol{z})-\boldsymbol{x}]^{\mathrm{T}}\right\} \geqslant \boldsymbol{J}_{\mathrm{B}}^{-1}=\boldsymbol{C}_{\mathrm{B}}
$$

where $\boldsymbol{C}_{\mathrm{B}}$ denotes the BCRB matrix, and $\boldsymbol{J}_{\mathrm{B}}$ is the $(i, j)$ th term of the Bayesian information matrix (BIM), which can be caculated by

$$
\boldsymbol{J}_{\mathrm{B}}[i, j]=\mathrm{E}_{\boldsymbol{z}, \boldsymbol{x}}\left\{-\frac{\partial^{2} \ln p(\boldsymbol{z}, \boldsymbol{x})}{\partial \boldsymbol{z}(i) \partial \boldsymbol{z}(j)}\right\}
$$

where $p(\boldsymbol{z}, \boldsymbol{x})$ is the joint probability density function of $(\boldsymbol{z}, \boldsymbol{x})$, and $\boldsymbol{z}(i)$ is the $i$ th element of $\boldsymbol{z}$. The equation (10) shows that the BCRB can quantify the range-only target tracking performance. The sequence of the posterior fish information matrices (FIMs) $\boldsymbol{J}_{\mathrm{B}}\left(\boldsymbol{x}_{k}\right), k>0$ is derived for the unbiased estimation of the target state $\boldsymbol{x}_{k}$ [18]

$$
\boldsymbol{J}_{\mathrm{B}}\left(\boldsymbol{x}_{k}\right)=\left[\boldsymbol{Q}_{k}+\boldsymbol{F} \boldsymbol{J}_{\mathrm{B}}^{-1}\left(\boldsymbol{x}_{k-1}\right) \boldsymbol{F}^{\mathrm{T}}\right]^{-1}+\boldsymbol{J}_{\mathrm{D}}\left(\boldsymbol{x}_{k}\right)
$$

where $\boldsymbol{J}_{\mathrm{D}}\left(\boldsymbol{x}_{k}\right)=\mathrm{E}_{\boldsymbol{x}_{k}}\left[\boldsymbol{J}_{\boldsymbol{r}}\left(\boldsymbol{x}_{k}\right)\right]$, and $\mathrm{E}_{\boldsymbol{x}_{k}}(\cdot)$ denotes the expectation of the target state $\boldsymbol{x}_{k}$. The matrix $\boldsymbol{J}_{\boldsymbol{r}}\left(\boldsymbol{x}_{k}\right)$ can be recast through the chain rule

$$
\boldsymbol{J}_{\boldsymbol{r}}\left(\boldsymbol{x}_{k}\right)=\boldsymbol{H}\left(\boldsymbol{x}_{k}\right) \cdot\left(\boldsymbol{C}_{z}^{k}\right)^{-1} \cdot \boldsymbol{H}^{\mathrm{T}}\left(\boldsymbol{x}_{k}\right)
$$

where Jacobin matrix $\boldsymbol{H}\left(\boldsymbol{x}_{k}\right)=\Delta_{\boldsymbol{x}_{k}}\left\{h\left(\boldsymbol{x}_{k}\right)\right\}, \Delta_{\boldsymbol{x}_{k}}\{\cdot\}$ denotes the derivation with $\boldsymbol{x}_{k}$. In this paper, the target location accuracy is focused on, and obviously,

$$
\boldsymbol{H}\left(\boldsymbol{x}_{k}\right)=\left[\begin{array}{c}
\cos \phi_{m}^{k}+\cos \varphi_{n}^{k} \\
\sin \phi_{m}^{k}+\sin \varphi_{n}^{k} \\
0 \\
0
\end{array}\right],
$$

so we can obtain

$$
\boldsymbol{J}_{\mathrm{D}}\left(\boldsymbol{x}_{k}\right)=\left[\begin{array}{cccc}
g_{k} & z_{k} & 0 & 0 \\
z_{k} & h_{k} & 0 & 0 \\
0 & 0 & 0 & 0 \\
0 & 0 & 0 & 0
\end{array}\right]
$$


where $g_{k}, h_{k}, z_{k}$ are respectively defined as follows [18]:

$$
\left\{\begin{array}{l}
g_{k}=\eta \sum_{m=1}^{M} v_{m}^{k} \mathrm{E}_{\boldsymbol{x}_{k}}\left[A_{m}^{k}\right] \\
h_{k}=\eta \sum_{m=1}^{M} v_{m}^{k} \mathrm{E}_{\boldsymbol{x}_{k}}\left[B_{m}^{k}\right] \\
z_{k}=\eta \sum_{m=1}^{M} v_{m}^{k} \mathrm{E}_{\boldsymbol{x}_{k}}\left[C_{m}^{k}\right]
\end{array}\right.
$$

where $\eta=\frac{8 \pi^{2}}{c^{2} \sigma_{\omega}^{2}}, v_{m}^{k}=u_{m}^{k} p_{m}^{k}\left(\beta_{m}^{k}\right)^{2}$, and the elements $A_{m}^{k}, B_{m}^{k}, C_{m}^{k}$ are defined [12] as

$$
\left\{\begin{aligned}
A_{m}^{k} & =\sum_{n=1}^{N} \alpha_{m n}\left|h_{m n}\right|^{2}\left(\cos \phi_{m}^{k}+\cos \varphi_{n}^{k}\right)^{2} \\
B_{m}^{k} & =\sum_{n=1}^{N} \alpha_{m n}\left|h_{m n}\right|^{2}\left(\sin \phi_{m}^{k}+\sin \varphi_{n}^{k}\right)^{2} \\
C_{m}^{k} & =\sum_{n=1}^{N} \alpha_{m n}\left|h_{m n}\right|^{2}\left(\sin \phi_{m}^{k}+\sin \varphi_{n}^{k}\right) \\
& \left(\cos \phi_{m}^{k}+\cos \varphi_{n}^{k}\right)
\end{aligned}\right.
$$

Since the expectation in (14) is a nonlinear function of $\boldsymbol{x}_{k}$, it is difficult to be solved analytically. In this case, the Monte Carlo approach may be alternative to obtain the result numerically. We assume that the initial target state $\boldsymbol{x}_{0}$ is subject to Gaussian distribution $N\left(\overline{\boldsymbol{x}}_{0}, \boldsymbol{P}_{0}\right)$ approximately, where $\boldsymbol{P}_{0}$ denotes the initial covariance matrix. Then the target state $\boldsymbol{x}_{k}$ is also subject to Gaussian distribution at any time $k$ from the target motion (4). Furthermore, the relationship of the mean value covariance matrix between time $k-1$ and time $k$ can be written as

$$
\left\{\begin{array}{l}
\overline{\boldsymbol{x}}_{k}=\boldsymbol{F} \overline{\boldsymbol{x}}_{k-1} \\
\boldsymbol{P}_{k}=\boldsymbol{F} \boldsymbol{P}_{k-1} \boldsymbol{F}^{\mathrm{T}}+\boldsymbol{Q}_{k}
\end{array} .\right.
$$

The mean value covariance matrix of the target state can be obtained at any time by (15). Furthermore, we can generate a large amount of virtual training samples of the target state $\boldsymbol{x}_{k}=\left(x_{k}, y_{k}, \dot{x}_{k}, \dot{y}_{k}\right)^{\mathrm{T}}$ at time $k$ according to $N\left(\overline{\boldsymbol{x}}_{k}, \boldsymbol{P}_{k}\right)$.

The MSE of the target location estimate is bounded by the BCRB

$$
\operatorname{var}\left(\widehat{x}^{k}\right)+\operatorname{var}\left(\widehat{y}^{k}\right) \geqslant\left[\boldsymbol{J}_{\mathrm{B}}^{-1}\left(\boldsymbol{x}_{k}\right)\right]_{11}+\left[\boldsymbol{J}_{\mathrm{B}}^{-1}\left(\boldsymbol{x}_{k}\right)\right]_{22}
$$

where $\left(\widehat{x}^{k}, \widehat{y}^{k}\right)$ denotes the target location estimation at time $k$ and $[\cdot]_{i j}$ denotes the $i j$ th element of the matrix. Noticing that the first item in (12) is a constant term at time $k$ [18], it can be disregarded. Then we may just focus on maximizing $\boldsymbol{J}_{\mathrm{D}}\left(\boldsymbol{x}_{k}\right)$. We denote the inverse of $\boldsymbol{J}_{\mathrm{D}}\left(\boldsymbol{x}_{k}\right)$ as $T\left(\boldsymbol{u}^{k}, \boldsymbol{p}^{k}, \boldsymbol{\beta}^{k}\right)$, which can be recast as

$$
T\left(\boldsymbol{u}^{k}, \boldsymbol{p}^{k}, \boldsymbol{\beta}^{k}\right)=\frac{\left(g_{k}+h_{k}\right)}{g_{k} h_{k}-z_{k}^{2}}=\frac{\left(\boldsymbol{A}^{k}+\boldsymbol{B}^{k}\right)^{\mathrm{T}} \boldsymbol{v}^{k}}{\left(\boldsymbol{v}^{k}\right)^{\mathrm{T}} \boldsymbol{H}^{k} \boldsymbol{v}^{k}}
$$

where $\boldsymbol{v}^{k}=\left[v_{1}^{k}, v_{2}^{k}, \ldots, v_{M}^{k}\right]^{\mathrm{T}}$ and $\boldsymbol{A}^{k}, \boldsymbol{B}^{k}, \boldsymbol{H}^{k}$ are respectively defined as

$$
\left\{\begin{array}{l}
\boldsymbol{A}^{k}=\eta\left[\mathrm{E}_{\boldsymbol{x}_{k}}\left\{A_{1}^{k}\right\}, \mathrm{E}_{\boldsymbol{x}_{k}}\left\{A_{2}^{k}\right\}, \ldots, \mathrm{E}_{\boldsymbol{x}_{k}}\left\{A_{M}^{k}\right\}\right]^{\mathrm{T}} \\
\boldsymbol{B}^{k}=\eta\left[\mathrm{E}_{\boldsymbol{x}_{k}}\left\{B_{1}^{k}\right\}, \mathrm{E}_{\boldsymbol{x}_{k}}\left\{B_{2}^{k}\right\}, \ldots, \mathrm{E}_{\boldsymbol{x}_{k}}\left\{B_{M}^{k}\right\}\right]^{\mathrm{T}} \\
\boldsymbol{C}^{k}=\eta\left[\mathrm{E}_{\boldsymbol{x}_{k}}\left\{C_{1}^{k}\right\}, \mathrm{E}_{\boldsymbol{x}_{k}}\left\{C_{2}^{k}\right\}, \ldots, \mathrm{E}_{\boldsymbol{x}_{k}}\left\{C_{M}^{k}\right\}\right]^{\mathrm{T}} \\
\boldsymbol{H}^{k}=\left[0.5 \boldsymbol{A}^{k}\left(\boldsymbol{B}^{k}\right)^{\mathrm{T}}+0.5 \boldsymbol{B}^{k}\left(\boldsymbol{A}^{k}\right)^{\mathrm{T}}-\boldsymbol{C}^{k}\left(\boldsymbol{C}^{k}\right)^{\mathrm{T}}\right]
\end{array} .\right.
$$

\section{Joint resource allocation scheme}

In the previous section, the BCRB for range-only target tracking is derived, which is a function of the number of transmit sensors, power and bandwidth. We aim at minimizing the BCRB by the joint sensor subset selection (SSS) and allocating resources among the selected sensors to achieve a better target tracking performance.

\subsection{Problem formulation}

The BCRB is minimized by the joint SSS and optimal power and bandwidth allocation (OPBA) under the predetermined resource constraint, and the optimization problem is formulated as

$$
\begin{gathered}
\min _{\boldsymbol{u}^{k}, \boldsymbol{p}^{k}, \boldsymbol{\beta}^{k}} T\left(\boldsymbol{u}^{k}, \boldsymbol{p}^{k}, \boldsymbol{\beta}^{k}\right) \\
\text { s.t. }\left\{\begin{array}{l}
\mathbf{1}^{\mathrm{T}} \boldsymbol{u}^{k}=L \\
\mathbf{1}^{\mathrm{T}} \boldsymbol{p}^{k}=P_{\text {total }} \\
\mathbf{1}^{\mathrm{T}} \boldsymbol{\beta}^{k}=B_{\text {total }} \\
u_{m}^{k} \in\{0,1\}, m \in\{M\} \\
p_{m_{\min }}^{k} \leqslant p_{m}^{k} \leqslant p_{m_{\max }}^{k}, m \in\{S\} \\
\beta_{m_{\min }}^{k} \leqslant \beta_{m}^{k} \leqslant \beta_{m_{\max }}^{k}, m \in\{S\} \\
p_{m}^{k}=\beta_{m}^{k}=0, m \in\{M \backslash S\}
\end{array}\right.
\end{gathered}
$$

where $S$ denotes a subset of $M . L$ denotes the number of the transmitter that the radar system chooses actually, and the reasonable choice of it can refer to $[22,23] . P_{\text {total }}$ and $B_{\text {total }}$ are the total power and bandwidth constraint. The minimum and maximum constraints on the power and bandwidth are defined in [17].

$$
\begin{aligned}
& p_{m_{\text {min }}}^{k}=0.02 P_{\text {total }}, \quad p_{m_{\text {max }}}^{k}=0.5 P_{\text {total }} \\
& \beta_{m_{\text {min }}}^{k}=0.02 B_{\text {total }}, \quad \beta_{m_{\text {max }}}^{k}=0.5 B_{\text {total }}
\end{aligned}
$$

The last constraint $p_{m}^{k}=\beta_{m}^{k}=0, m \in\{M \backslash S\}$ denotes that the power and bandwidth among the unselected sensors is zero. Since there is a Boolean constraint in the conditions, and the matrix $\boldsymbol{H}^{k}$ in the objective function is neither positive semi-definite nor negative semi-definite, the optimization problem is non-convex-Boolean, and an approach is needed to solve it. In general, most of these 
techniques cannot obtain the global optimum, and their complexity grows exponentially when the dimension increases. The SPCA is taken into consideration, which is firstly mentioned in [24]. Its core idea is to extract the concave part from the non-convex constraints, and transform the problem into an approximate one by Taylor expansion [25].

Since there are three variable vectors involved in this joint optimization problem, it needs to be simplified firstly. Proposition 1 is introduced to reveal the relationship between the optimal power allocation vector and the bandwidth allocation vector.

Proposition 1 If the sensor selection result is disregarded temporarily, there is

$$
\beta_{j}^{k}=\frac{B_{\text {total }}}{P_{\text {total }}} p_{j}^{k}
$$

where $\beta_{j}^{k}$ and $p_{j}^{k}$ denote the $j$ th elements of the bandwidth allocation vector $\boldsymbol{\beta}^{k}$ and the optimal power allocation vector $\boldsymbol{p}^{k}$, respectively.

Proof by contradiction is used. If the sensor selection result is disregarded temporarily, new power and bandwidth allocation vectors $\left(\widehat{\boldsymbol{p}}^{k}, \widehat{\boldsymbol{\beta}}^{k}\right)$ are defined, of which the $j$ th element is

$$
\left\{\begin{array}{l}
\widehat{p}_{j}^{k}=\frac{P_{\text {total }}}{\sum_{j=1}^{M}\left(p_{j}^{k}\right)^{\frac{1}{3}}\left(\beta_{j}^{k}\right)^{\frac{2}{3}}}\left(p_{j}^{k}\right)^{\frac{1}{3}}\left(\beta_{j}^{k}\right)^{\frac{2}{3}} \\
\widehat{\beta}_{j}^{k}=\frac{B_{\text {total }}}{\sum_{j=1}^{M}\left(p_{j}^{k}\right)^{\frac{1}{3}}\left(\beta_{j}^{k}\right)^{\frac{2}{3}}}\left(p_{j}^{k}\right)^{\frac{1}{3}}\left(\beta_{j}^{k}\right)^{\frac{2}{3}}
\end{array}\right.
$$

where $P_{\text {total }}$ and $B_{\text {total }}$ are collinear. Take new solutions into the objective function in (20), then

$$
T\left(\widehat{\boldsymbol{p}}^{k}, \widehat{\boldsymbol{\beta}}^{k}\right)=\frac{\left[\sum_{j=1}^{M}\left(p_{j}^{k}\right)^{\frac{1}{3}}\left(\beta_{j}^{k}\right)^{\frac{2}{3}}\right]^{3}}{P_{\text {total }} B_{\text {total }}^{2}} T\left(\boldsymbol{p}^{k}, \boldsymbol{\beta}^{k}\right) .
$$

Since $p_{j}^{k}$ and $\beta_{j}^{k}$ are both greater than zero and $\mathbf{1}^{\mathrm{T}} \boldsymbol{p}^{k}=$ $P_{\text {total }}, \mathbf{1}^{\mathrm{T}} \boldsymbol{\beta}^{k}=B_{\text {total }}$, we can deduce the following equation from Holder's equation:

$$
\begin{gathered}
T\left(\widehat{\boldsymbol{p}}^{k}, \widehat{\boldsymbol{\beta}}^{k}\right)=\frac{\left[\sum_{j=1}^{M}\left(p_{j}^{k}\right)^{\frac{1}{3}}\left(\beta_{j}^{k}\right)^{\frac{2}{3}}\right]^{3}}{P_{\text {total }} B_{\text {total }}^{2}} T\left(\boldsymbol{p}^{k}, \boldsymbol{\beta}^{k}\right) \leqslant \\
\frac{\left[\left(\mathbf{1}^{\mathrm{T}} \boldsymbol{p}^{k}\right)^{\frac{1}{3}}\left(\mathbf{1}^{\mathrm{T}} \boldsymbol{\beta}^{k}\right)^{\frac{2}{3}}\right]^{3}}{P_{\text {total }} B_{\text {total }}^{2}} T\left(\boldsymbol{p}^{k}, \boldsymbol{\beta}^{k}\right)=T\left(\boldsymbol{p}^{k}, \boldsymbol{\beta}^{k}\right)
\end{gathered}
$$

where we can find that there is always a new and better allocation strategy $\left(\widehat{\boldsymbol{p}}^{k}, \widehat{\boldsymbol{\beta}}^{k}\right)$ satisfying the condition $\widehat{\beta}_{j}^{k}=\frac{B_{\text {total }}}{P_{\text {total }}} \widehat{p}_{j}^{k}$, which is superior to the assumed optimal solution $\left(\boldsymbol{p}^{k}, \boldsymbol{\beta}^{k}\right)$. Thus, it is not difficult to learn that $\left(\boldsymbol{p}^{k}, \boldsymbol{\beta}^{k}\right)$ has to satisfy $\beta_{j}^{k}=\frac{B_{\text {total }}}{P_{\text {total }}} p_{j}^{k}$ at the same time.

The optimization problem can be simplified with two independent vectors $\left(\boldsymbol{p}^{k}, \boldsymbol{u}^{k}\right)$ by Proposition 1.

\subsection{Optimization scheme}

Assuming that $\delta$ is the upper bound of the objective, the optimization problem can be formulated as

$$
\begin{aligned}
& \min _{\boldsymbol{u}^{k}, \boldsymbol{p}^{k}} \delta \\
& \text { s.t. }\left\{\begin{array}{l}
\left(\boldsymbol{A}^{k}+\boldsymbol{B}^{k}\right)^{\mathrm{T}} \boldsymbol{v}^{k}-\delta\left(\boldsymbol{v}^{k}\right)^{\mathrm{T}} \boldsymbol{H}^{k} \boldsymbol{v}^{k} \leqslant 0 \\
\boldsymbol{v}^{k}=\boldsymbol{u}^{k} \odot\left[\left(\frac{B_{\text {total }}}{P_{\text {total }}}\right)^{2}\left(\boldsymbol{p}^{k}\right)^{3}\right] \\
\mathbf{1}^{\mathrm{T}} \boldsymbol{u}^{k}=L \\
\mathbf{1}^{\mathrm{T}} \boldsymbol{p}^{k}=P_{\text {total }} \\
u_{m}^{k} \in\{0,1\}, \quad m \in\{M\} \\
p_{m_{\min }}^{k} \leqslant p_{m}^{k} \leqslant p_{m_{\max }}^{k}, \quad m \in\{S\} \\
p_{m}^{k}=0, \quad m \in\{M \backslash S\}
\end{array}\right.
\end{aligned}
$$

According to the SPCA approach, we need to extract the concave part of the non-convex constraints about $\boldsymbol{H}^{k}$ elaborated firstly in (21). An alternative approach is to decompose $\boldsymbol{H}^{k}$ into a non-negative definite matrix $\left(\boldsymbol{H}^{k}\right)^{+}$ and a non-positive definite matrix $\left(\boldsymbol{H}^{k}\right)^{-}$.

$$
\begin{gathered}
\left(\boldsymbol{A}^{k}+\boldsymbol{B}^{k}\right)^{\mathrm{T}} \boldsymbol{v}^{k}-\delta\left(\boldsymbol{v}^{k}\right)^{\mathrm{T}}\left(\boldsymbol{H}^{k}\right)^{-} \boldsymbol{v}^{k}- \\
\delta\left(\boldsymbol{v}^{k}\right)^{\mathrm{T}}\left(\boldsymbol{H}^{k}\right)^{+} \boldsymbol{v}^{k} \leqslant 0 .
\end{gathered}
$$

Then the concave parts $-\left(\boldsymbol{v}^{k}\right)^{\mathrm{T}}\left(\boldsymbol{H}^{k}\right)^{+} \boldsymbol{v}^{k}$ can be converted into a convex problem by linearizing around the solution $\boldsymbol{v}_{i}^{k}$, where $\boldsymbol{v}_{i}^{k}=\boldsymbol{u}_{i}^{k} \odot\left(\frac{B_{\text {total }}}{P_{\text {total }}}\right)^{2}\left(\boldsymbol{p}_{i}^{k}\right)^{3}$ and $i$ indexes the iteration. The linearization can be implemented by a first order Taylor expansion, where

$$
\begin{gathered}
\left(\boldsymbol{A}^{k}+\boldsymbol{B}^{k}\right)^{\mathrm{T}} \boldsymbol{v}^{k}-\delta\left(\boldsymbol{v}^{k}\right)^{\mathrm{T}}\left(\boldsymbol{H}^{k}\right)^{-} \boldsymbol{v}^{k}- \\
\delta\left(\boldsymbol{v}_{i}^{k}\right)^{\mathrm{T}}\left(\boldsymbol{H}^{k}\right)^{+}\left(2 \boldsymbol{v}^{k}-\boldsymbol{v}_{i}^{k}\right) \leqslant 0 .
\end{gathered}
$$

The constraint of $\boldsymbol{H}^{k}$ in (23) is convex, and its solution is a subset of the solution in (22). Several iterations are required. The uniform allocation is set as the initialization point, which is equal to or worse than the final solution. Set the solution of the previous iteration as the linearization point to the next one, and keep iterating until the objective is not improved anymore. Then the optimization problem can be separated into four steps and solved by the cyclic minimization algorithm. 
Step 1 Obtain the convex relaxation of the SSS problem by replacing $u_{m}^{k} \in\{0,1\}$ with $0 \leqslant u_{m}^{k} \leqslant 1$ [12]. Set the initial sensor subset $\widehat{\boldsymbol{u}}_{\mathrm{opt}}^{k}$ randomly.

Step 2 Minimize the BCRB for a given power budge. We can also minimize the BCRB for a given sensor number firstly, with the following steps reversed. Assume $\delta$ to be a constant and introduce the following proposition.

Proposition 2 The solution $\boldsymbol{p}^{k}$ to the following new problem,

$$
\begin{gathered}
\min _{\boldsymbol{p}^{k}} \mathbf{1}^{\mathrm{T}} \boldsymbol{p}^{k} \\
\text { s.t. }\left\{\begin{array}{c}
\left(\boldsymbol{A}^{k}+\boldsymbol{B}^{k}\right)^{\mathrm{T}} \boldsymbol{v}^{k}-\delta\left(\boldsymbol{v}^{k}\right)^{\mathrm{T}}\left(\boldsymbol{H}^{k}\right)^{-} \boldsymbol{v}^{k}- \\
\delta\left(\boldsymbol{v}_{i}^{k}\right)^{\mathrm{T}}\left(\boldsymbol{H}^{k}\right)^{+}\left(2 \boldsymbol{v}^{k}-\boldsymbol{v}_{i}^{k}\right) \leqslant 0 \\
\boldsymbol{v}^{k}=\widehat{\boldsymbol{u}}_{\mathrm{opt}}^{k} \odot\left(\frac{B_{\text {total }}}{P_{\text {total }}}\right)^{2}\left(\boldsymbol{p}^{k}\right)^{3} \\
p_{m_{\min }}^{k} \leqslant p_{m}^{k} \leqslant p_{m_{\max }}^{k}
\end{array}\right.
\end{gathered}
$$

is collinear with the optimal solution $\boldsymbol{p}_{\mathrm{opt}}^{k}$ to the original problem (27) by

$$
\boldsymbol{p}_{\mathrm{opt}}^{k}=\frac{P_{\mathrm{total}}}{\mathbf{1}^{\mathrm{T}} \boldsymbol{p}^{k}} \boldsymbol{p}^{k}
$$

The proof is also by contradiction. Denoting the optimal solution $\boldsymbol{p}_{\mathrm{opt}}^{k}$ to the origin optimization problem (21), the optimal solution to problem (27) is $\boldsymbol{p}^{k}$. Assume that $\boldsymbol{p}_{\text {opt }}^{k}$ is a more optimal solution compared with $\alpha \boldsymbol{p}^{k}$, where $\alpha$ is a constant satisfying $0 \leqslant \alpha \leqslant \frac{P_{\text {total }}}{\mathbf{1}^{\mathrm{T}} \boldsymbol{p}^{k}}$. Considering the maximum value, i.e., $\alpha=\frac{P_{\text {total }}}{\mathbf{1}^{\mathrm{T}} \boldsymbol{p}^{k}}$, it is obvious that $\mathbf{1}^{\mathrm{T}}\left(\alpha \boldsymbol{p}^{k}\right)=P_{\text {total }}$, which satisfies the total power constraint. According to the assumption, the following equation is satisfied:

$T\left(\boldsymbol{p}_{\text {opt }}^{k}\right)<T\left(\alpha \boldsymbol{p}^{k}\right)=\left(\frac{\mathbf{1}^{\mathrm{T}} \boldsymbol{p}^{k}}{P_{\text {total }}}\right)^{3} \cdot T\left(\boldsymbol{p}^{k}\right) \leqslant \delta \cdot\left(\frac{\mathbf{1}^{\mathrm{T}} \boldsymbol{p}^{k}}{P_{\text {total }}}\right)^{3}$

where $\boldsymbol{p}_{\mathrm{opt}}^{k}$ is the optimal solution to the problem (27) such that it also satisfies the constraint $\mathbf{1}^{\mathrm{T}} \boldsymbol{p}_{\mathrm{opt}}^{k}=P_{\text {total }}$. Then, defining a new power allocation vector $\boldsymbol{p}^{*}=$ $\sqrt[3]{T\left(\boldsymbol{p}_{\mathrm{opt}}^{k}\right) / \delta} \boldsymbol{p}_{\mathrm{opt}}^{k}$, we compute the sum with respect to $\boldsymbol{p}^{*}$ :

$$
\mathbf{1}^{\mathrm{T}} \boldsymbol{p}^{*}=\sqrt[3]{\frac{T\left(\boldsymbol{p}_{\mathrm{opt}}^{k}\right)}{\delta}} \mathbf{1}^{\mathrm{T}} \boldsymbol{p}_{\mathrm{opt}}^{k}<\left(\frac{\mathbf{1}^{\mathrm{T}} \boldsymbol{p}^{k}}{P_{\text {total }}}\right) \mathbf{1}^{\mathrm{T}} \boldsymbol{p}_{\mathrm{opt}}^{k}=\mathbf{1}^{\mathrm{T}} \boldsymbol{p}^{k} .
$$

The equation (33) shows that the new solution $p^{*}$ is a better solution than $\boldsymbol{p}^{k}$ to the problem (21), which contradicts with the original assumption. Thus, the solution $\boldsymbol{p}^{k}$ satisfies $\boldsymbol{p}^{k} \propto \boldsymbol{p}_{\mathrm{opt}}^{k}$, and the relationship between $\boldsymbol{p}^{k}$ and $\boldsymbol{p}_{\mathrm{opt}}^{k}$ is obtained by $\mathbf{1}^{\mathrm{T}} \boldsymbol{p}_{\mathrm{opt}}^{k}=P_{\mathrm{total}}$ :

$$
\boldsymbol{p}_{\mathrm{opt}}^{k}=\frac{P_{\text {total }}}{\mathbf{1}^{\mathrm{T}} \boldsymbol{p}^{k}} \boldsymbol{p}^{k} .
$$

For the power allocation vector $\boldsymbol{p}^{k}$ is the sixth order in the second constraint of (27), a slack vector $\boldsymbol{z}^{k}=$ $\left[z_{1}^{k}, \ldots, z_{M}^{k}\right]^{\mathrm{T}}$ is introduced, which is related with $\boldsymbol{p}^{k}$ by $z_{m}^{k}=\left(p_{m}^{k}\right)^{3}$. We relax this constraint by $z_{m}^{k}-\left(p_{m}^{k}\right)^{3} \leqslant 0$, the optimal solution must satisfy this relaxed constraint with equality. Reduction to absurdity is used here, but it has been omitted to simplify the description.

$$
\begin{gathered}
\min _{\boldsymbol{z}^{k}, \boldsymbol{p}^{k}} \mathbf{1}^{\mathrm{T}} \boldsymbol{p}^{k} \\
\text { s.t. }\left\{\begin{array}{c}
\left(\boldsymbol{A}^{k}+\boldsymbol{B}^{k}\right)^{\mathrm{T}} \boldsymbol{v}^{k}-\delta\left(\boldsymbol{v}^{k}\right)^{\mathrm{T}}\left(\boldsymbol{H}^{k}\right)^{-} \boldsymbol{v}^{k}- \\
\delta\left(\boldsymbol{v}_{i}^{k}\right)^{\mathrm{T}}\left(\boldsymbol{H}^{k}\right)^{+}\left(2 \boldsymbol{v}^{k}-\boldsymbol{v}_{i}^{k}\right) \leqslant 0 \\
\boldsymbol{v}^{k}=\widehat{\boldsymbol{u}}_{\mathrm{opt}}^{k} \odot\left(\frac{B_{\text {total }}}{P_{\text {total }}}\right)^{2} \boldsymbol{z}^{k} \\
z_{m}^{k}-\left(p_{m}^{k}\right)^{3} \leqslant 0 \\
p_{m_{\min }}^{k} \leqslant p_{m}^{k} \leqslant p_{m_{\max }}^{k}
\end{array}\right.
\end{gathered}
$$

According to the SPCA approach, we can also convexify the concave part $-\left(p_{m}^{k}\right)^{3}$ by linearizing this part around the point $p_{m, i}^{k}$, which is the $m$ th element of the $\boldsymbol{p}_{i}^{k}$ that satisfies $\boldsymbol{v}_{i}^{k}=\widehat{\boldsymbol{u}}_{\mathrm{opt}}^{k} \odot\left(\frac{B_{\text {total }}}{P_{\text {total }}}\right)^{2}\left(\boldsymbol{p}_{i}^{k}\right)^{3}$, where $i$ indexes the number of iteration. The problem can be rewritten as

$$
\begin{gathered}
\min _{\boldsymbol{z}^{k}, \boldsymbol{p}^{k}} \mathbf{1}^{\mathrm{T}} \boldsymbol{p}^{k} \\
\text { s.t. }\left\{\begin{array}{l}
\left(\boldsymbol{A}^{k}+\boldsymbol{B}^{k}\right)^{\mathrm{T}} \boldsymbol{v}^{k}-\delta\left(\boldsymbol{v}^{k}\right)^{\mathrm{T}}\left(\boldsymbol{H}^{k}\right)^{-} \boldsymbol{v}^{k}- \\
\delta\left(\boldsymbol{v}_{i}^{k}\right)^{\mathrm{T}}\left(\boldsymbol{H}^{k}\right)^{+}\left(2 \boldsymbol{v}^{k}-\boldsymbol{v}_{i}^{k}\right) \leqslant 0 \\
z_{m}^{k}+2\left(p_{m, i}^{k}\right)^{3}-3\left(p_{m, i}^{k}\right)^{3} p_{m}^{k} \leqslant 0, \quad m \in\{M\} \\
\boldsymbol{v}^{k}=\widehat{\boldsymbol{u}}_{\mathrm{opt}}^{k} \odot\left(\frac{B_{\text {total }}}{P_{\text {total }}}\right)^{2} \boldsymbol{z}^{k} \\
p_{m_{\min }}^{k} \leqslant p_{m}^{k} \leqslant p_{m_{\max }}^{k}
\end{array}\right.
\end{gathered}
$$

It can be solved by the SPCA algorithm effectively, and then $\widehat{\boldsymbol{p}}^{k}$ can be obtained. Based on $\widehat{\boldsymbol{p}}^{k}$ and (28), a suboptimal $\widehat{\boldsymbol{p}}_{\text {opt }}^{k}$ is obtained.

Step 3 Minimize the BCRB for a given sensor number. After $\widehat{\boldsymbol{p}}_{\mathrm{opt}}^{k}$ is obtained, the optimization problem in (27) can be expressed as

$$
\begin{gathered}
\min _{\boldsymbol{u}^{k}} \delta \\
\text { s.t. }\left\{\begin{array}{c}
\left(\boldsymbol{A}^{k}+\boldsymbol{B}^{k}\right)^{\mathrm{T}} \boldsymbol{v}^{k}-\delta\left(\boldsymbol{v}^{k}\right)^{\mathrm{T}}\left(\boldsymbol{H}^{k}\right)^{-} \boldsymbol{v}^{k}- \\
\delta\left(\boldsymbol{v}_{i}^{k}\right)^{\mathrm{T}}\left(\boldsymbol{H}^{k}\right)^{+}\left(2 \boldsymbol{v}^{k}-\boldsymbol{v}_{i}^{k}\right) \leqslant 0 \\
0 \leqslant u_{m}^{k} \leqslant 1, \quad m \in\{M\}
\end{array}\right.
\end{gathered}
$$


where $\boldsymbol{v}^{k}=\boldsymbol{u}^{k} \odot\left[\left(\frac{B_{\text {total }}}{P_{\text {total }}}\right)^{2}\left(\widehat{\boldsymbol{p}}_{\text {opt }}^{k}\right)^{3}\right]$. It can be solved by the SPCA algorithm combined with the convex optimization, and then a new suboptimal $\widehat{\boldsymbol{u}}_{\mathrm{opt}}^{k}$ is obtained.

Step 4 Keep returning to Step 2 until (21) is not improved anymore. Denote $\boldsymbol{u}_{\mathrm{opt}}^{k}$ as the optimal solution to the optimization problem, which can be obtained in the last iteration. Replace the largest element of $\boldsymbol{u}_{\mathrm{opt}}^{k}$ with one, and the others with zero. When the subset selection $S$ is known, the optimal power solution $\boldsymbol{p}_{\mathrm{opt}}^{k}$ can be obtained by solving (27). The optimal bandwidth allocation $\boldsymbol{\beta}_{\mathrm{opt}}^{k}$ can be generated by (20).

\section{Simulation results}

Assume $M=8$ and $N=4$ in the system, and the predetermined size of the transmit sensor subset is $L=4$. Two different static sensors geometrical deployments are given to analyze their influence on range-only target tracking, which are shown in Fig. 2. The carrier frequency $f_{c}=9 \mathrm{GHz}$, and the signal noise variance $\sigma_{\omega}^{2}=0.01$.

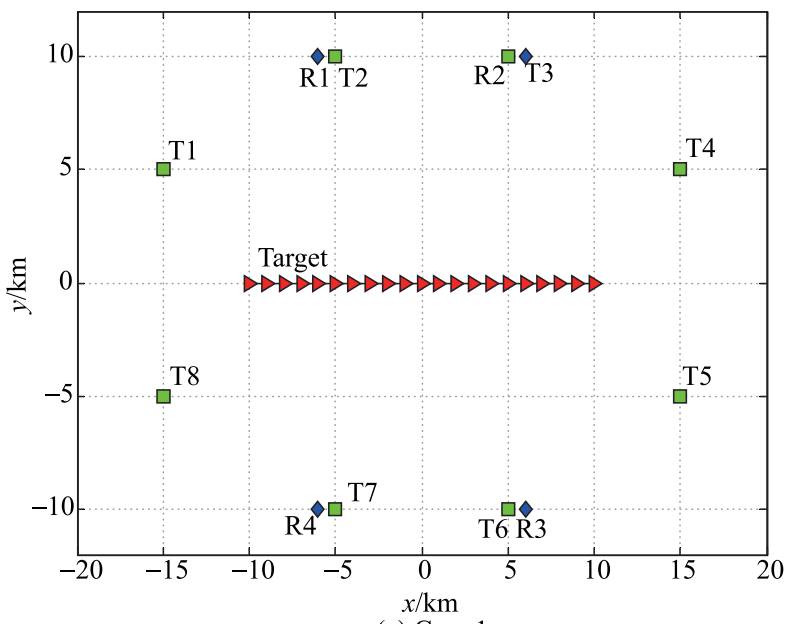

(a) Case 1

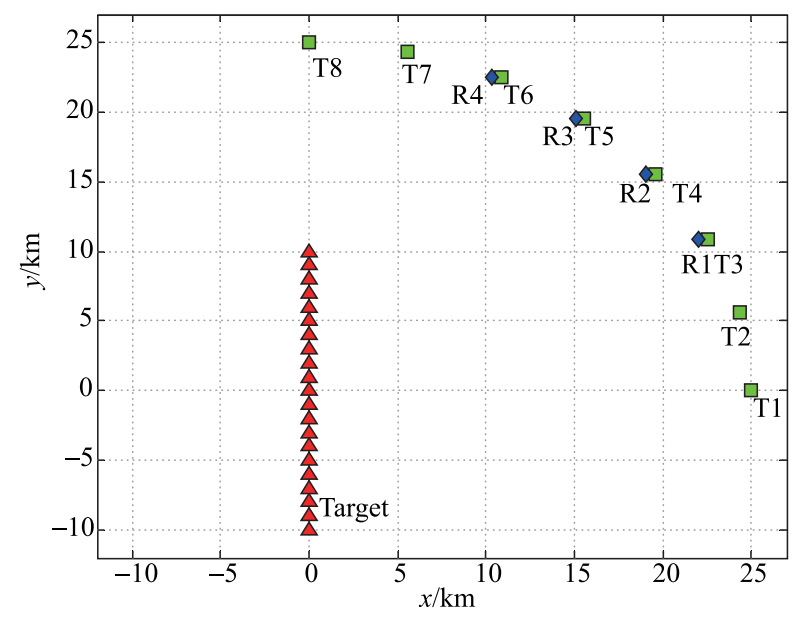

(b) Case 2

Fig. 2 Two distributed MIMO radar configurations
Assume that there are 21 frames data for the simulation, and the observation interval is $\Delta t=5 \mathrm{~s}$. The total power and bandwidth budgets are $P_{\text {total }}=10^{6} \mathrm{~W}$ and $B_{\text {total }}=10 \mathrm{MHz}$, respectively. Two target RCS models are considered: the first model is uniform reflectivity, i.e., $\boldsymbol{h}_{1}=[1, \ldots, 1 ; 1, \ldots, 1]$, which is to evaluate the impact of different system geometric topologies. In the second RCS model $\boldsymbol{h}_{2}$, the reflectivities of T4 and T5 are lower than the other sensors, which are the same as the first RCS model, as depicted in Fig. 3. The prior targets in two configurations are following Gaussian distribution with mean $\overline{\boldsymbol{x}}_{0}(1)=(-10 \mathrm{e} 3,0,200,0)^{\mathrm{T}} \mathrm{m}$ and $\overline{\boldsymbol{x}}_{0}(2)=(5 \mathrm{e} 3,0,200,0)^{\mathrm{T}} \mathrm{m}$.

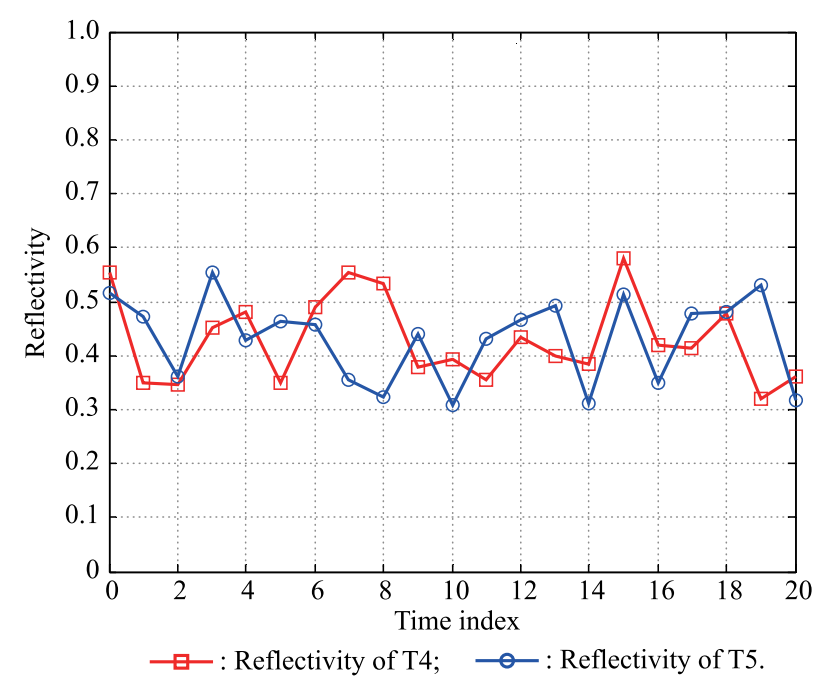

Fig. 3 The second RCS model $h_{2}$

In this section, we investigate the resource allocation of each sensor firstly. The percentages of the allocated resources are calculated based on the total amount of the power and bandwidth. Fig. 4 depicts the results of allocation with the uniform reflectivity $\boldsymbol{h}_{1}$. It is worth noting that the sensor subset selection results vary from time to time and case to case. In case 1, T1, T2, T7 and T8 are superior to the others in location. As the target moves, $\mathrm{T} 1$ and $\mathrm{T} 8$ are replaced by $\mathrm{T} 3$ and $\mathrm{T} 6$. The resource are allocated to the sensors closer to the moving target at a better angle. From Fig. 4(b), the system resource will give priority to better sensors as the target moves towards T8. It shows that $\mathrm{T} 1$ is the closest transmit sensor in the beginning, while $\mathrm{T} 8$ is the closest to the last position of the target.

Fig. 5 depicts the resource of each transmitter for different sensor geometries with $\boldsymbol{h}_{2}$. As we can observe that in Fig. 5(a), T4 and T5 are selected at the 17th time index, as opposed to the 16th time index in Fig. 4(a). Besides, the resource of T4 and T5 in Fig. 5(a) is lower than that in Fig. 4(a). This situation also appears in Case 2 with $\boldsymbol{h}_{2}$, as depicted in Fig. 5(b). The above results demonstrate 
that the top four transmit sensors are selected at any time and the resource gives priority to the closer sensors with a larger RCS.

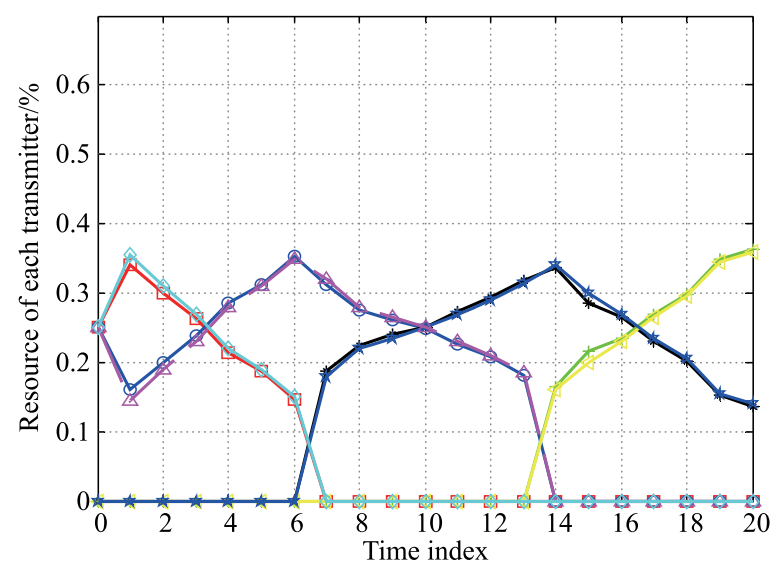

(a) Case 1

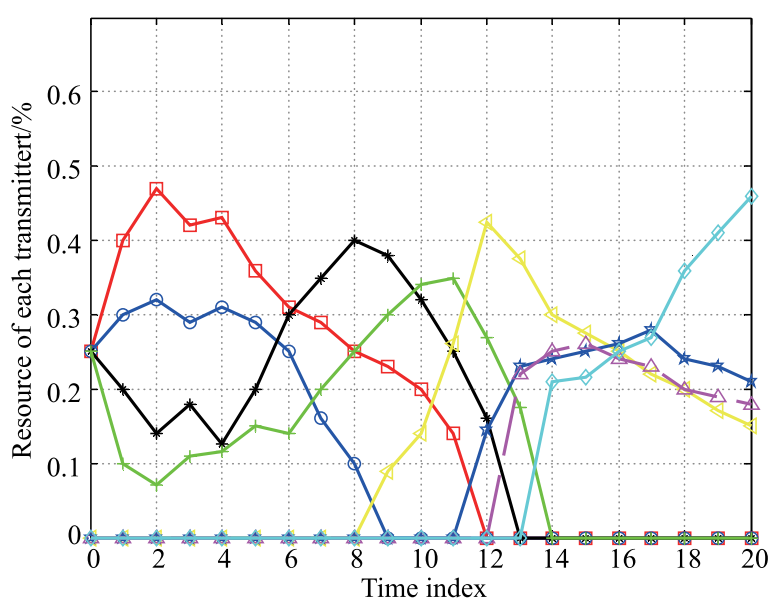

(b) Case 2

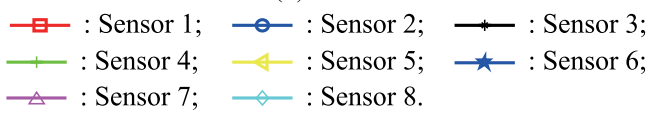

Fig. 4 Resource allocation results with $h_{1}$

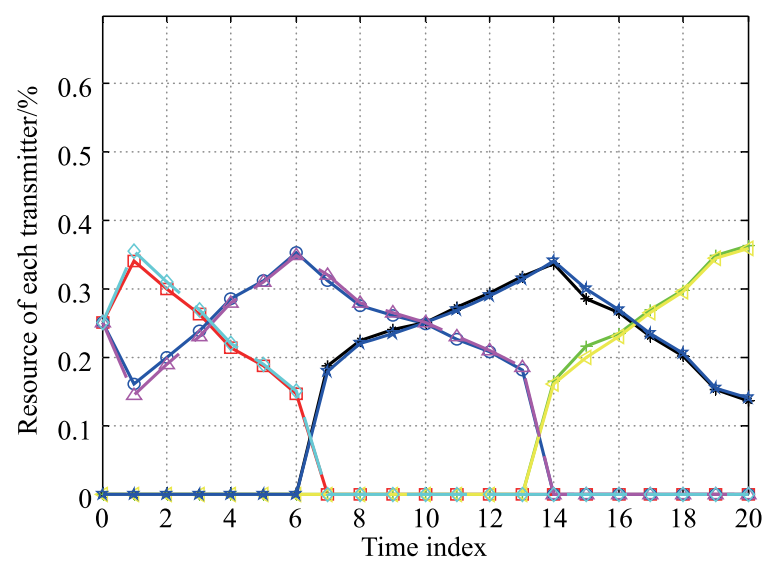

(a) Case 1

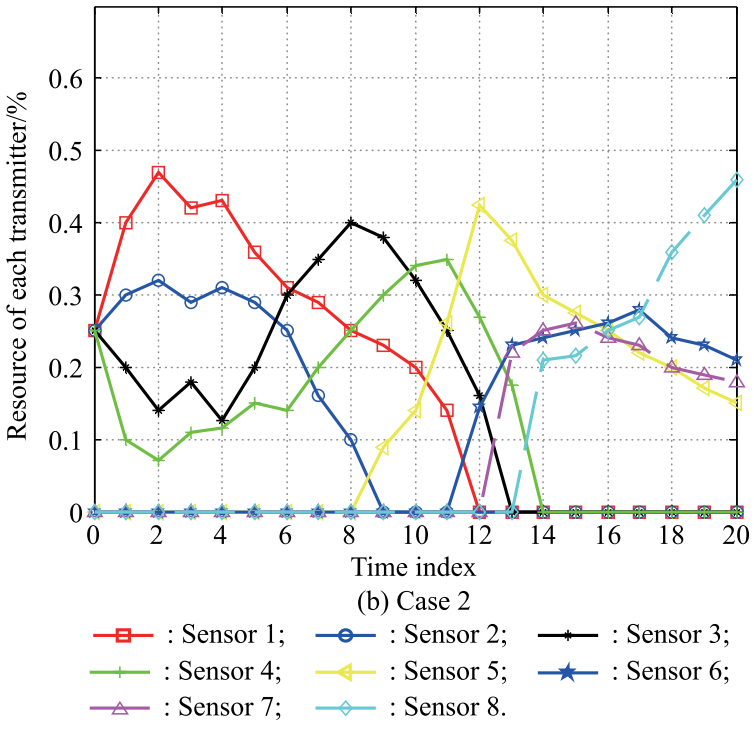

Fig. 5 Resource allocation results with $h_{2}$

Then, there is a comparison between the BCRB of the target tracking accuracy with different MIMO radar topologies and two RCS models in Fig. 6 and Fig. 7. We compare the performance of the proposed scheme (OPBA with SSS) with other resource allocation schemes including uniform resource allocation (URA), single SSS case [22], optimal power allocation (OPA) with SSS method and optimal bandwidth allocation (OBA) with SSS method, respectively.

For two MIMO radar topologies with different RCS models, the results show that the proposed scheme can improve the target tracking accuracy significantly, while the URA without the SSS scheme is the worst. The OBA with the SSS method is the second only to the optimal one, while the OPA with the SSS method is a little bit better than the URA with the SSS method, except for Case 1 where the target is located at the origin of coordinates at the 11th time index.

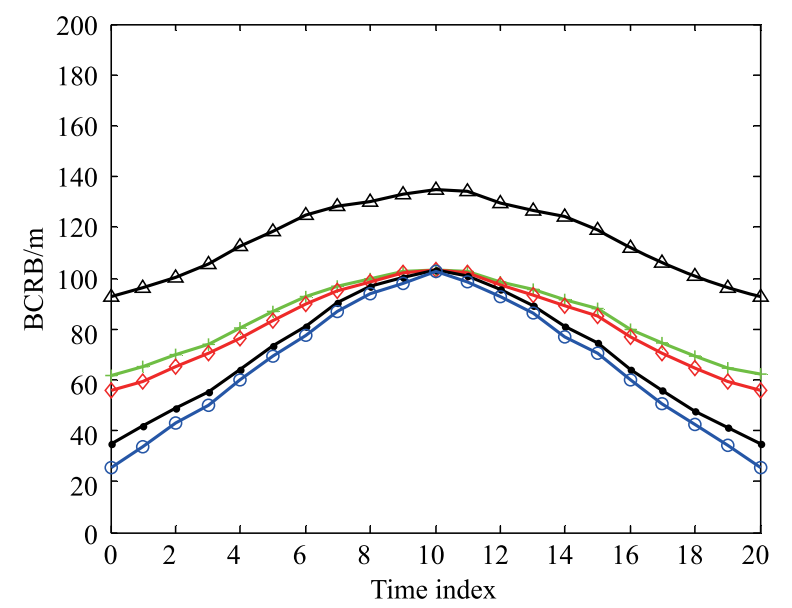

(a) Case 1 


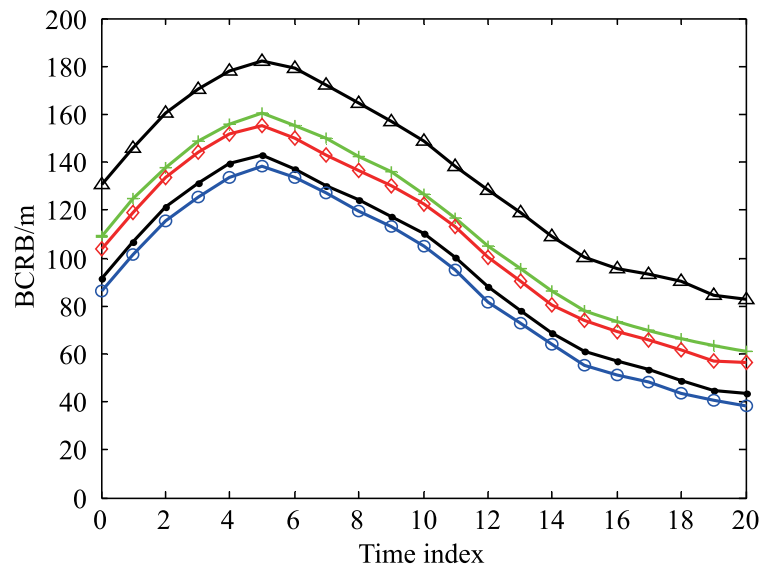

(b) Case 2

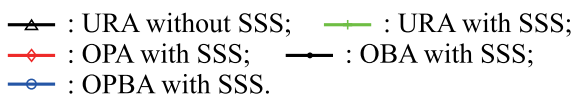

Fig. 6 BCRB of target tracking with $h_{1}$

It is also observed from Fig. 7 that, OPBA with the SSS scheme reduces the tracking errors by almost two thirds with URA using all sensors when the RCS model is $\boldsymbol{h}_{2}$. Furthermore, Fig. 6 and Fig. 7 illustrate that the bandwidth plays a more prominent role than power in rangeonly target tracking, for the bandwidth allocation vector is a quadratic variable, while the power allocation vector is of the first order in (20).

On the other hand, to validate the effectiveness of the target tracking method, localization errors are also taken into consideration in the simulation process. At first, we apply the WRELAX algorithm [26] to estimate the time of arrivals (TOA) of the transmitted signals at the receivers. It is followed by estimating the target position by using the BLUE method as shown in [7]. The square root of the localization error in two distributed MIMO radar configurations with $\boldsymbol{h}_{1}$ is shown in Fig. 8. We can draw a similar conclusion that the proposed resource allocation method can reduce the target location errors.

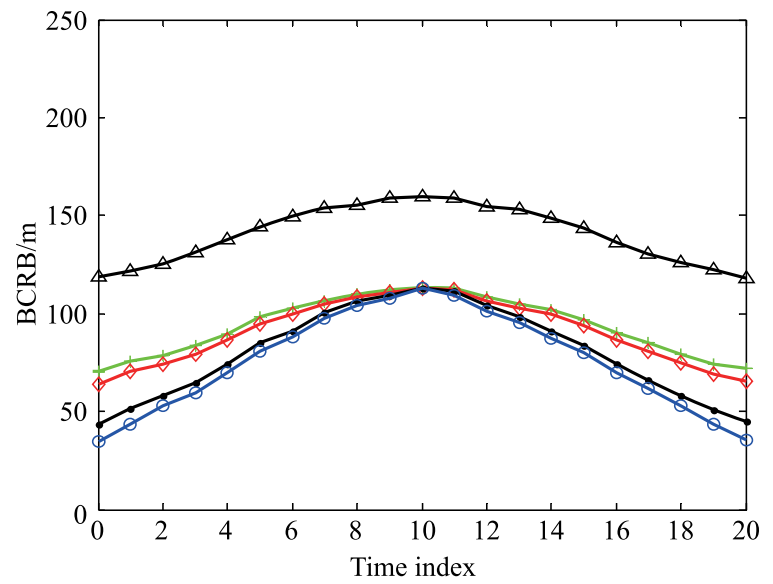

(a) Case 1

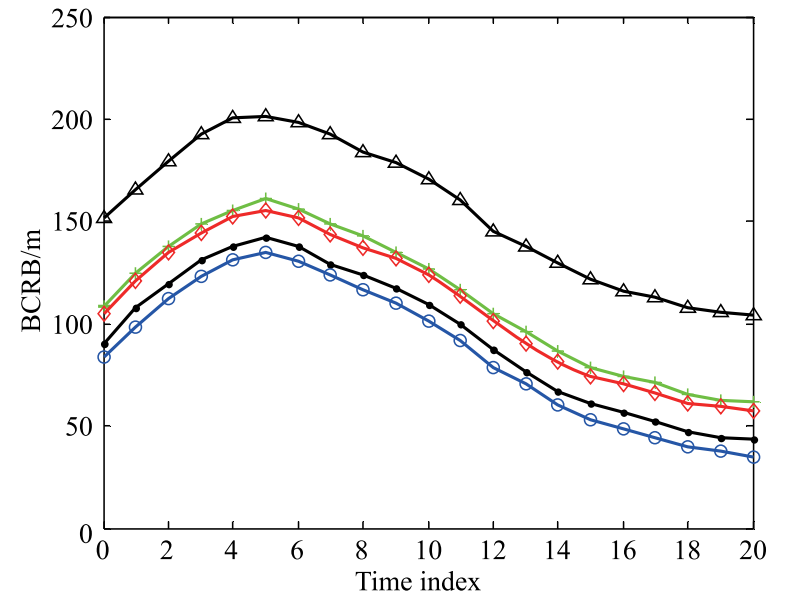

(b) Case 2

$\triangle$ : URA without SSS; $\quad \longrightarrow$ : URA with SSS;

$\neg$ : OPA with SSS; $\rightarrow:$ OBA with SSS;

$\rightarrow$ : OPBA with SSS.

Fig. 7 BCRB of target tracking with $h_{2}$

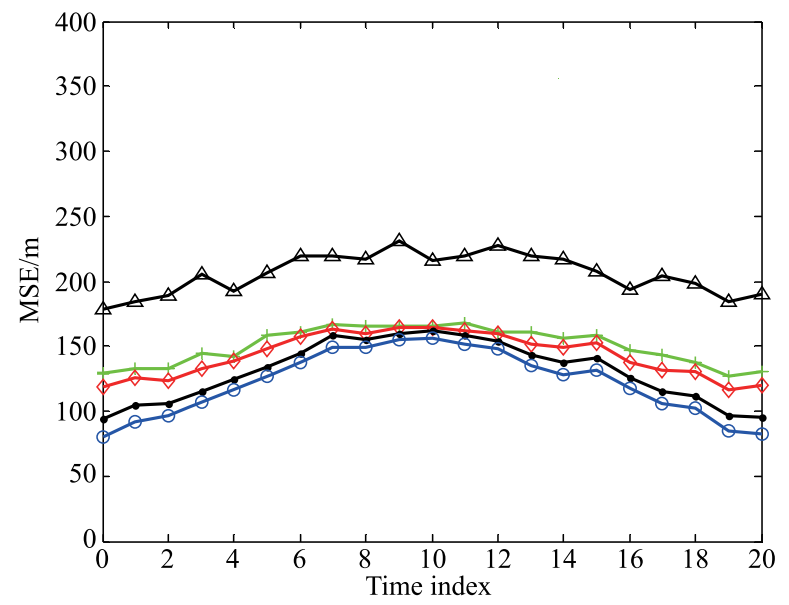

(a) Case 1

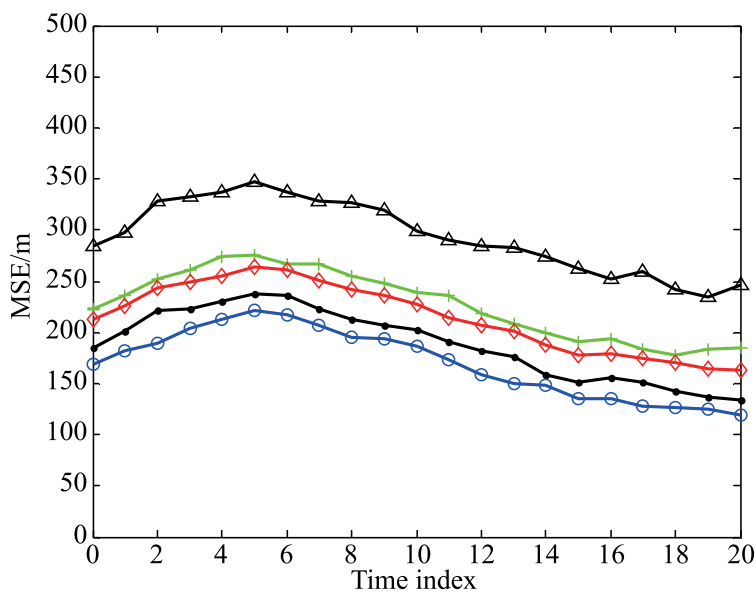

(b) Case 2

$\rightarrow$ : URA without SSS; — : URA with SSS $\neg$ : OPA with SSS; $\rightarrow$ : OBA with SSS $\rightarrow$ : OPBA with SSS

Fig. 8 Square root of the localization error in two distributed MIMO radar configurations with $h_{1}$ 
Finally, to make simulation results more convincing, the Monte Carlo simulation method is employed. In each simulation, the transmitters and receivers are positioned randomly in a $20 \times 20 \mathrm{~km}^{2}$ area, according to a uniform distribution. The target motion model is the same as in Case 1 and its RCS model is $\boldsymbol{h}_{1}$. The results of the resource allocation are evaluated from the average of 500 values from (19). Fig.9 plots the BCRB and the square root of errors in the localization of the target. The result validates the effectiveness of the target tracking method in different layouts.

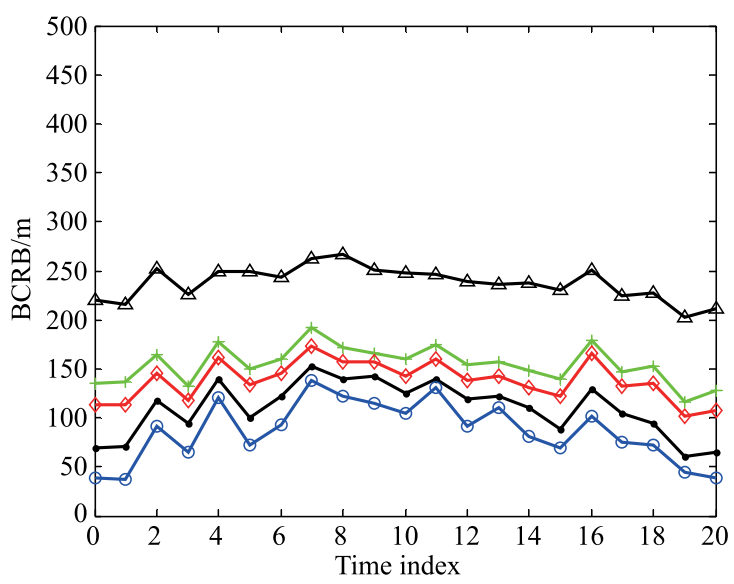

(a) Case 1

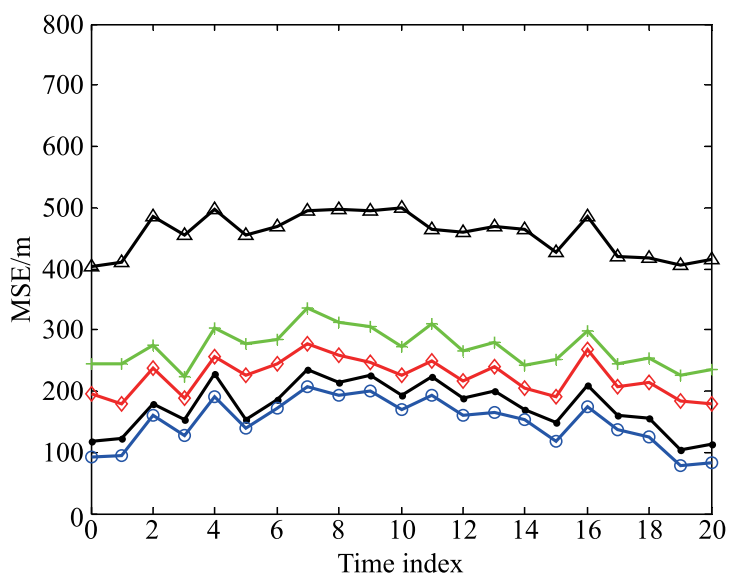

(b) Case 2

$\triangle$ : URA without SSS; - : URA with SSS;

$\rightarrow$ : OPA with SSS; $\longrightarrow$ : OBA with SSS

$\rightarrow$ : OPBA with SSS

Fig. 9 BCRB and square root of the localization $\left(h_{1}\right)$

\section{Conclusions}

The research on the MIMO radar is developed rapidly this decade. However, the issue about the resource management has not been emphasized adequately. To achieve a better range-only target tracking performance in distributed MIMO radar systems, a jonit resource allocation scheme called OPBA with the SSS scheme is presented.
The three-variable optimization problem is simplified by deriving the relationship between the optimal power allocation vector and the bandwidth allocation vector. Then, the simplified problem is solved by the cyclic minimization algorithm incorporating the SPCA algorithm. The simulation results reveal that the resource allocation strategy is related to the system topologies and the target RCS. Moreover, the proposed scheme outperforms the URA without the SSS method obviously, and the bandwidth plays a more important role than the power in range-only target tracking. It is certain that the reasonable resource allocation policy plays an important role for the better system performance with MIMO radar in the near future.

\section{References}

[1] MOHAMMAD M C, MOJTABA R, MOHAMMAD N M, et al. Improving MIMO radar's performance through receivers' positioning. IET Signal Processing, 2017, 11(5): 622-630.

[2] LI J, STOICA P. MIMO radar with colocated antennas. IEEE Signal Processing Magazine, 2007, 24(5): 106 - 114.

[3] CHEN H W, LI X, ZHUANG Z. A rising radar system-MIMO radar. Acta Electronica Sinica, 2012, 40(6): 1190-1198. (in Chinese)

[4] HAIMOVICH A M, BLUM R S, CIMINI L J. MIMO radar with widely separated antennas. IEEE Signal Processing Magazine, 2008, 25(1): 116-129.

[5] LI J, STOICA P, XU L, et al. On parameter identifiability of MIMO radar. IEEE Signal Processing Letters, 2007, 14(12): $968-971$.

[6] GODRICH H, HAIMOVICH A M, BLUM R S. Target localization accuracy gain in MIMO radar-based systems. IEEE Trans. on Information Theory, 2010, 56(6): 2783-2803.

[7] GODRICH H, CHIRIAC V, HAIMOVICH A M, et al. Target tracking in MIMO radar systems: techniques and performance analysis. Proc. of the IEEE Radar Conference, 2010: $1111-$ 1116.

[8] CHEN Y, ZHANG Q, LUO Y. Multi-target radar imaging based on phased-MIMO technique-part II: adaptive resource allocation. IEEE Sensors Journal, 2017, 17(19): 6198-6209.

[9] ANASTASIOS D, ANASTASIA P, SANGARAPILLAI L. Game-theoretic power allocation and the Nash equilibrium analysis for a multistatic MIMO radar network. IEEE Trans. on Signal Processing, 2017, 65(24): 6397-6408.

[10] GAO L Y, ZHENG N E, SONG X Y. Review on resource allocation in MIMO radar. Proc. of the IEEE International Conference on Computer \& Communications, 2017: 1-6.

[11] YAN J K, PU W, LIU H. Robust chance constrained power allocation scheme for multiple target localization in colocated MIMO radar system. IEEE Trans. on Signal Processing, 2018, 66(15): 3946-3957.

[12] GODRICH H, PETROPULU A, POOR H V. Cluster allocation schemes for target tracking in multiple radar architectures. Proc. of Signals, Systems and Computers Conference, 2011: $863-867$.

[13] SONG X Y, ZHENG N E, BAI T. Resource allocation schemes for multiple targets tracking in distributed MIMO radar systems. International Journal of Antennas \& Propagation, 2017, 
10: $1-12$.

[14] GODRICH H, PETROPULU A, POOR H V. Resource allocation schemes for target localization in distributed multiple radar architectures. Proc. of the European Signal Processing Conference, 2010: 23-27.

[15] GODRICH H, PETROPULU A, POOR H V. Power allocation strategies for target localization in distributed multiple-radar architecture. IEEE Trans. on Signal Processing, 2011, 59(7): $3226-3240$.

[16] GARCIA N, HAIMOVICH A, COULON M, et al. Resource allocation in MIMO radar with multiple targets for noncoherent localization. IEEE Trans. on Signal Processing, 2014, 62(10): 2656-2666.

[17] MA B, CHEN H, SUN B, et al. A joint scheme of antenna selection and power allocation for localization in MIMO radar sensor networks. IEEE Communications Letters, 2014, 18(12): $2225-2228$.

[18] CHEN H, TA S, SUN B. Cooperative game approach to power allocation for target tracking in distributed MIMO radar sensor networks. IEEE Sensors Journal, 2015, 15(10): 5423-5432.

[19] HU H Y, SUN Y, ZHENG N E. Resource allocation approach in distributed MIMO radar with multiple targets for velocity estimation. Journal of Electronics \& Information Technology, 2016, 38(10): 2453 - 2460. (in Chinese)

[20] WANG L, ZENG Y. Jamming power allocation strategy for MIMO radar based on MMSE and mutual information. IET Radar Sonar \& Navigation, 2017, 11(7): 1081 - 1089.

[21] BECK A, BENTAL A, TETRUASHVILI L. A sequential parametric convex approximation method with applications to nonconvex truss topology design problems. Journal of Global Optimization, 2010, 47(1): 29-51.

[22] DAI W H, SHEN Y, WIN M Z. On the minimum number of active anchors for optimal localization. Proc. of the IEEE Global Communication Conference, 2012: 4951 - 4956.

[23] DAI W H, SHEN Y, WIN M Z. Sparsity-inspired power allocation for network localization. Proc. of the IEEE International Conference Communication, 2012: 2785-2790.

[24] MARKS B R, WRIGHT G P. A general inner approximation algorithm for nonconvex mathematical problems. Journal of Operational Research, 1978, 26(4): 681-683.

[25] ASPREMONT A, BOYD S. Relaxations and randomized methods for nonconvex QCQPs. EE392o Class Notes, Stanford University, 2013.

[26] LI J, WU R. An efficient algorithm for time delay estimation. IEEE Trans. on Signal Processing, 1998, 46(8): 2231 - 2235.

\section{Biographies}

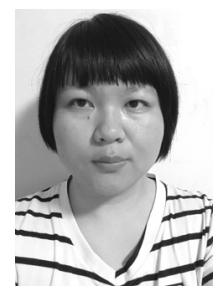

ZHENG Na'e was born in 1984. She received her B.S. degree in electronic engineering from $\mathrm{Na}-$ tional Digital Switching System Engineering and Technological R\&D Center (NDSC), Zhengzhou, China, in 2007, M.S. degree in communication and information system from NDSC in 2010, and Ph.D. degree in information and communication engineering from NDSC in 2014. Currently, she is a lecturer of PLA Strategic Support Force Information Engineering University. Her research interests include MIMO radar signal processing and analysis of fine features.

E-mail: zhengnaecshz@163.com

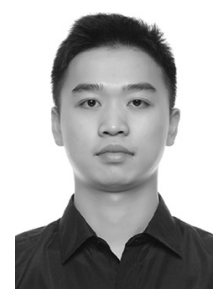

SUN Yang was born in 1992. He received his B.S. and M.S. degrees in electronic engineering from National Digital Switching System Engineering and Technological R\&D Center (NDSC) in 2014 and 2017, respectively. Now, he is a Ph.D. candidate in information and communication engineering of PLA Strategic Support Force Information Engineering University. His research interests include MIMO radar signal processing and image processing.

E-mail: sunyang_kd@163.com

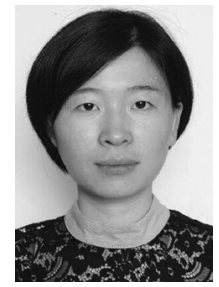

SONG Xiyu was born in 1992. She received her B.S. and M.S. degrees in electronic engineering from National Digital Switching System Engineering and Technological R\&D Center (NDSC) in 2015 and 2018, respectively. Now she is a Ph.D. candidate in information and communication engineering of PLA Strategic Support Force Information Engineering University. Her research interests include MIMO radar technology and life science.

E-mail: fsongxiyu@126.com

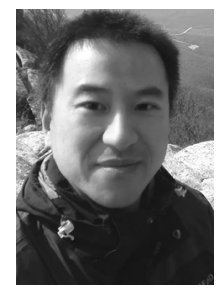

CHEN Song was born in 1986. He received his B.S. degree in electronic engineering from National Digital Switching System Engineering and Technological R\&D Center (NDSC) in 2008, M.S. degree in communication and information system from NDSC in 2011, and Ph.D. degree in information and communication engineering from NDSC in 2015. Currently, he is a lecturer of PLA Strategic Support Force Information Engineering University. His research interests include cognitive radio and MIMO radar systems.

E-mail: wirelessman@163.com 